\title{
Novelty Killing Public Disclosure
}

\section{Dylan Roskams-Edris ${ }^{1}$}

1 McGill University

Potential competing interests: The author(s) declared that no potential competing interests exist.

When information about an unpatented invention is disclosed publicly such that it would no longer meet the novelty requirement in patent law. 